\title{
Intra and extraarticular localized pigmented villonodular synovitis
}

\author{
Johney Juneja ${ }^{1 *}$, Raghvendra Choubisa ${ }^{1}$, Ramesh Sen ${ }^{2}$, \\ A. K. Mehra ${ }^{3}$, Basant Aggarwal ${ }^{3}$, Krishan Kumar Sharma ${ }^{3}$
}

\author{
${ }^{1}$ Orthopaedic Department, RNT Hospital, Udaipur, India \\ ${ }^{2}$ Max Hospital, Mohali, India \\ ${ }^{3}$ RNT Hospital, Udaipur, India
}

Received: 31 August 2021

Revised: 12 October 2021

Accepted: 14 October 2021

\author{
*Correspondence: \\ Johney Juneja, \\ E-mail: johney.johney2008@gmail.com
}

Copyright: () the author(s), publisher and licensee Medip Academy. This is an open-access article distributed under the terms of the Creative Commons Attribution Non-Commercial License, which permits unrestricted non-commercial use, distribution, and reproduction in any medium, provided the original work is properly cited.

\begin{abstract}
A 28 year old woman had a history of knee trauma and presented with unilateral knee acute swelling and pain symptoms with sudden onset, which was there from last 2 years. She had been treated for seronegative rheumatoid patient for 1 year. Recent expansion of the LPVNS (localized pigmented villonodular synovitis) caused the development of a tender palpable soft tissue mass in the anterolateral aspect of the knee and acute reduced mobility. Preoperative magnetic resonance imaging of the knee revealed the presence of only the soft tissue mass and mild degenerative changes. Open synovectomy was performed successfully to excise the mass. Intraoperatively, macroscopic features of the bright brown inflamed synovium suggested LPVNS, which was confirmed histopathologically. Postoperatively, the symptoms of limited mobility and pain were appreciably relieved. Recurrence was not observed during the clinical follow up at 1, 6 or 18 months after surgery. Here, we reported the unique case of localized pigmented villonodular synovitis of the knee in a misdiagnosed patient with intra and extraarticular lesion, which might be attributed to the history of knee trauma and the focal defect of the lateral patellar retinaculum. Open synovectomy effectively relieved the symptoms of limited mobility and pain and no recurrence was observed prior to 18 months postoperatively. To reduce misdiagnosis, MRI examinations are recommended for all patients suspected of having PVNS, including those who have a history of hyperuricemia.
\end{abstract}

Keywords: Case report, Pigmented villonodular synovitis, Intraarticular, Extraarticular, Synovectomy

\section{INTRODUCTION}

Pigmented villonodular synovitis (PVNS), also known as pigmented villonodular bursitis and pigmented villonodular tenosynovitis, is a benign, proliferative disease that occurs at the synovial membrane of joints, bursas, and tendon sheaths. There are two distinct forms: diffuse (DPVNS) and localized (LPVNS) pigmented villonodular synovitis. ${ }^{1}$ The diffuse form affects the whole synovium of the joint, while the localized form is characterized by focal involvement of the synovium. ${ }^{2}$ The localized form is usually a single pedunculated mass or less frequently, two or three nodules. Finding multiple nodules in LPVNS is extremely rare. To the best of our knowledge, there were only two reported cases. ${ }^{3}$ Here, we described a case of the multiple nodular form of LPVNS in the knee joint. It should be included as an atypical form of LPVNS in the differential diagnosis of knee problems. Although the exact pathogenesis of PVNS remained unclear, this disease was presumably closely related to trauma, intraarticular bleeding and inflammation. ${ }^{13}$ Due to the highly variable clinical presentation of PVNS, an accurate diagnosis was extremely difficult to achieve. Advanced imaging modalities such as magnetic resonance imaging (MRI) were considered the most sensitive approaches for the assessment of suspected PVNS. Soft 
masses with low signal intensity and blooming artifact on MRI scans appeared to be pathognomonic of PVNS. ${ }^{1,14,15}$ However, histopathology remained the gold standard tool to confirm the diagnosis. Surgical treatment for PVNS is considered as the standard treatment for these lesions, conservative treatments such as radiation therapy were also available. An in-depth understanding of the extent of the disease was needed to select the most appropriate treatment as the two types of PVNS required different treatments. Currently, PVNS was classified as diffuse (DPVNS) or localized (LPVNS). ${ }^{16}$ The two types of PVNS can be further categorized as intraarticular or extraarticular. Despite the histological similarity, there were differences between the types in clinical presentation, prognosis and response to treatment. ${ }^{17,18}$

Here, we described a patient who had massive intraarticular LPVNS with an extraarticular extension through the lateral patellar retinaculum; the lesion was completely excised in open synovectomy.

\section{CASE REPORT}

A 28 year old woman had a history of knee trauma and presented with unilateral knee acute swelling and pain symptoms with sudden onset, which was there from last 2 years.
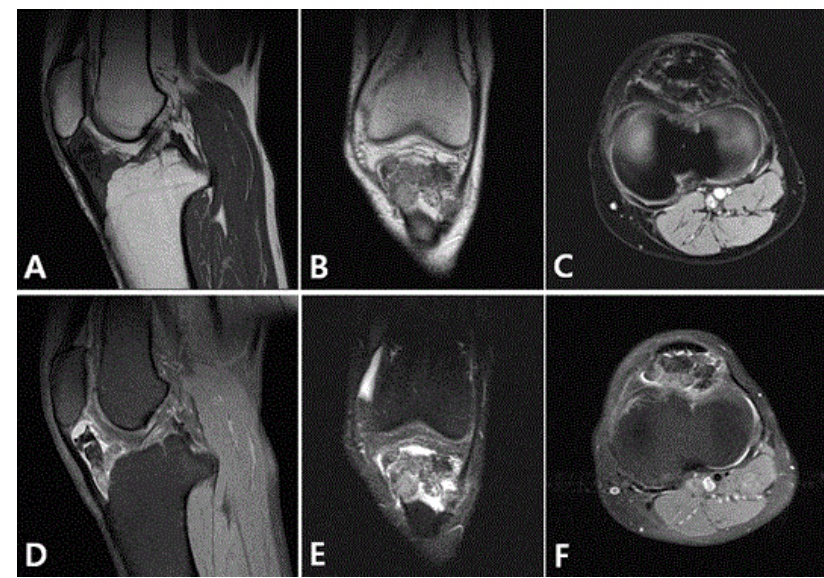

Figure 1: Preoperative MRI of the left knee shows multiple well-demarcated heterogeneous masses, with intermediate to low signal intensity on T1 and T2weighted images, surrounded by a fluid signal in the anterior compartment of the knee joint; (A) T1weighted sagittal; (B) T1-weighted coronal; (C) T1weighted axial; (D) T2-weighted sagittal; (E) T2weighted coronal; (F) T2-weighted axial.

She had been treated for seronegative rheumatoid patient for 1 year. Recent expansion of the LPVNS caused the development of a tender palpable soft tissue mass in the anterolateral aspect of the knee and acute reduced mobility.

She complained of recurrent swelling and pain in the knee, which may have been misdiagnosed as gouty arthritis at the local community hospital because of similar symptoms. The advised treatment based on the symptoms including DMARDS, colchicine and nonsteroidal antiinflammatory drugs, partially relieved the patient's symptoms.

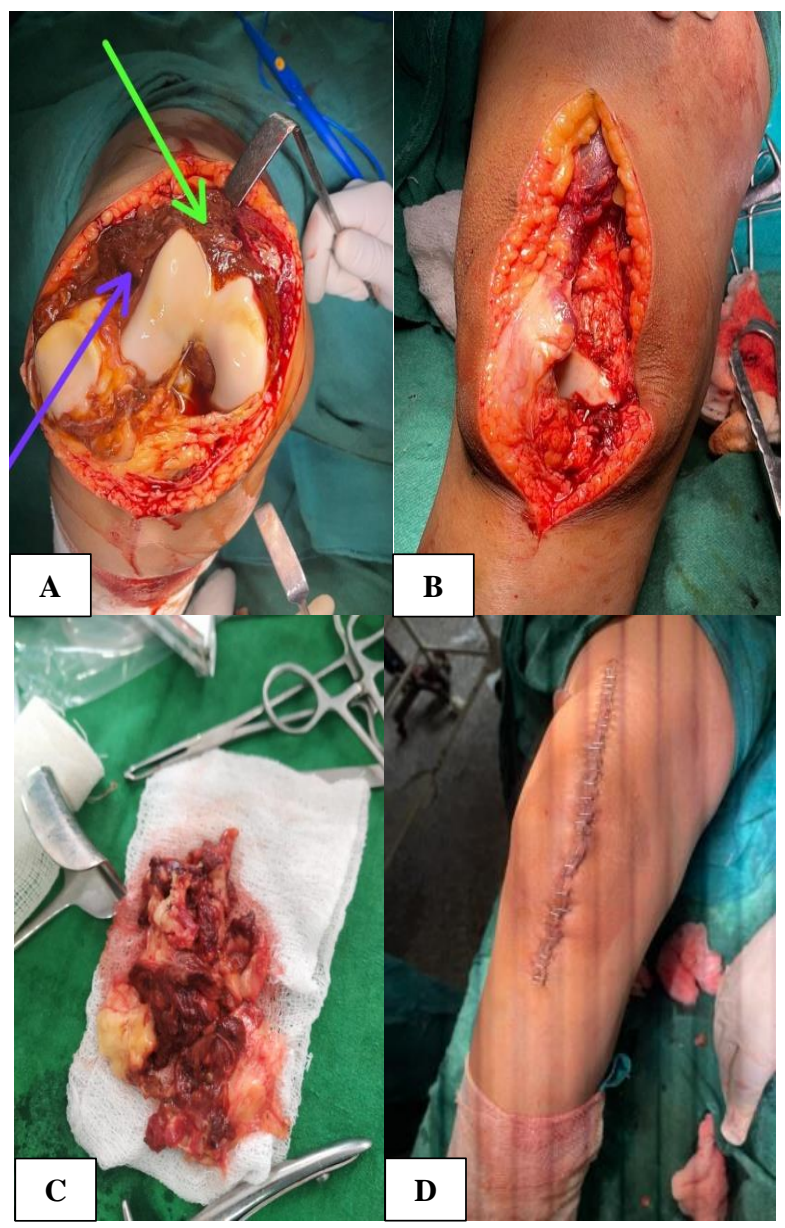

Figure 2: Intraoperative findings; (A) exposure of the extra-articular mass; ( $\mathrm{B}$ and $\mathrm{C}$ ) as the abnormal synovium was excised completely, the focal defect lateral patellar retinaculum was repaired; (D) the 13 $\mathrm{cm}$ median incision was sutured with skin staples.

The physical examination showed moderate, recurrent swelling of the left knee without surrounding warmth as well as a tender mass growing slowly in the anteroinferior aspect of the left knee. Because of pain, the patient's ROM in flexion had decreased considerably $\left(30-70^{\circ}\right.$ in the left knee, compared with $0-110^{\circ}$ in the right knee).

The results of the laboratory tests for both trioxypurine and infection were within the respective normal limits.

The MRI examination of the left knee revealed a massive intraarticular soft tissue lesion (measuring 58×32×46 mm) that replaced the normal Hoffa fat pad and extended through the lateral patellar retinaculum (Figure $1 \mathrm{~A}$ and $\mathrm{C}$ ). Moreover, the lobulated mass had a well circumscribed margin and exhibited focal heterogeneous hypo intensity representing the blooming artifact from hemosiderin, 
which was regarded as the pathognomonic appearance of PVNS on MRI (Figure 1B). Only mild degenerative changes and joint effusion were observed, without tophus or bone erosion.

Based on the MRI findings, it was considered impossible to completely remove the mass lesion. Therefore, open excision was selected as the surgical treatment. A $13 \mathrm{~cm}$ midline incision was made and synovectomy was performed successfully.

A bright brown inflamed lobulated synovium was observed intraoperatively (Figure 2A). Neither bone erosion nor diffuse soft tissue lesions were observed, suggesting a diagnosis of LPVNS.

The final diagnosis was further confirmed by postoperative histological analysis, the results of which were consistent with LPVNS, multinucleated giant cells with no atypical features and an abundance of macrophages loaded with hemosiderin.

Postoperatively, the patient was satisfied with the substantial improvement of knee ROM $\left(5-100^{\circ}\right)$. At the 1 , 6 and 18 month follow ups, the patient had no pain and exhibited full restoration of knee ROM, with neither complications nor radiological recurrence (Figure 3 ).

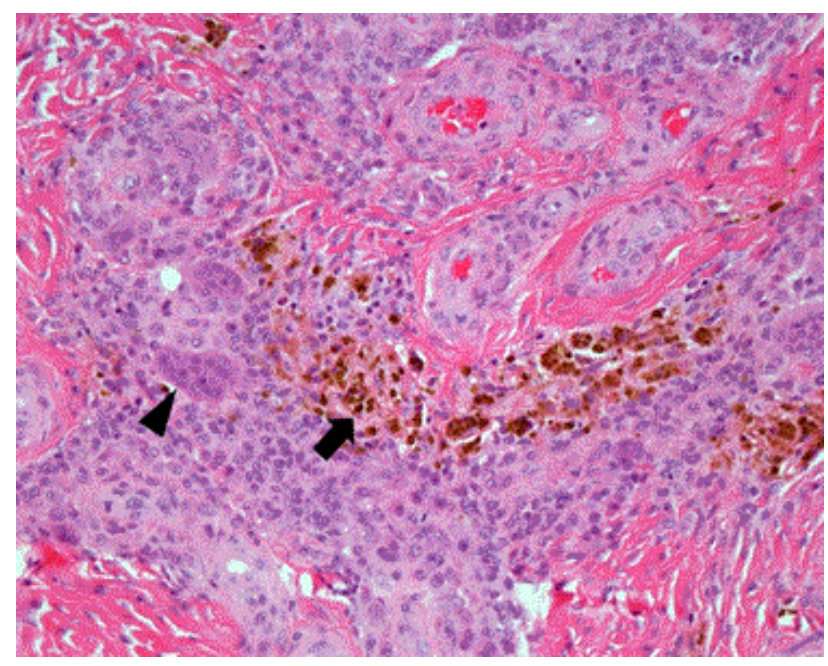

Figure 3: Pathological findings show the nodules surrounded by fibrous septa and foci of hemosiderinladen macrophages (arrow) and infiltration of polymorphic inflammatory cells with mononuclear histiocytes, some of which were forming multinucleated giant cells (arrowhead), (hematoxylin and eosin staining: original magnification $\times 200$ ).

\section{DISCUSSION}

The incidence of PVNS was $\sim 1.8$ cases per million people. ${ }^{4}$ The etiology of PVNS was still uncertain, chronic inflammation, trauma, hemarthrosis, neoplastic origin and chromosomal disorders have been considered. ${ }^{5}$ The most widely accepted theories implicated a chronic inflammatory process or a benign neoplasia. ${ }^{6}$ PVNS usually involved monoarticular joints commonly, the knee joint. However, cases in the hip, ankle, shoulder, elbow and even the temporomandibular joint have also been reported. ${ }^{4}$ PVNS was usually diagnosed in patients between the third and fourth decades. It did not seem to have any gender predisposition. ${ }^{4}$ The clinical presentation was diverse and the diagnosis was often delayed because patients were usually asymptomatic. The most effective diagnostic modality was MRI. ${ }^{7}$ Hemosiderin deposition in PVNS presented a low signal on $\mathrm{T} 1$ and $\mathrm{T} 2$ weighted images. Areas of bright signal on T2 weighted images may be present due to abnormal synovial membranes and joint fluid in the synovial membrane. ${ }^{8}$

There were two types of PVNS: diffuse and localized. ${ }^{9}$ Histological findings were similar but they were distinct disease entities for prognosis and treatment. The diffuse type comprising $75 \%$ of PVNS cases, involved most of the synovium with accompanying joint effusion. ${ }^{10}$ However, the localized form arose from certain parts of the synovium and resided within joints in a nodular form. The localized form was usually a single mass of pedunculated or less frequently, sessile appearance that was yellow-brown in color. However, two or three multilobulated localized masses and a mixed form, with a prominent mass and diffuse synovitis, representing a transition between DPVNS and LPVNS have also been reported. ${ }^{11,12}$ In our case, the multiple nodular form of PVNS was confined to the patellar fat pad, in a localized manner. Because the patient in this case had suffered from his symptoms for $\sim 20$ years, we assumed that nodules had increased in number over a long time and finally presented as a multiple lesion. To the best of our knowledge, there was only one report of a multinodular form of LPVNS, referred to as clustered LPVNS. ${ }^{3}$

Histologically, the circumscribed nodule was characterized by the active proliferation of fibroblastic and histiocytic elements showing evident macrophagic activity with phagocytosis of abundant blood pigment (hemosiderin) and lipids. Those elements were intermingled with a variable number of multinucleated giant cells and with a certain number of inflammatory elements, predominantly lympho-plasmocytes. ${ }^{9}$ Our case showed similar findings.

In treating PVNS, the purpose was to remove all abnormal synovial tissue, to prevent the risk of joint destruction and to avoid local recurrence. For the knee joint, several options have been suggested, from observation to total knee arthroplasty and including external beam radiation, radioactive synovectomy, surgical synovectomy, targeted therapy, immunotherapy and combinations of them. ${ }^{13} \mathrm{~A}$ recent systematic review reported no difference in local recurrence rates after open or arthroscopic surgery for LPVNS or DPVNS.

Recurrence rates of PVNS increased with time. Verspoor et al reported that $15 \%$ of recurrences occurred within the 
first year and $22 \%$ within 5 years in LPVNS. ${ }^{13}$ They also reported that the 1 and 5 year recurrence free survival rates for DPVNS were $69 \%$ and $32 \%$, respectively. In the present case, after arthroscopic resection, there was no recurrence at 3 years postoperatively.

In our case, the patient presented with long-lasting knee discomfort and mild swelling versus the contralateral knee. However, the range of motion was normal and no sign of instability, giving way or blocking was seen. The patient had received just roentgenography, not special imaging, such as MRI for $\sim 20$ years. Thus, we suspected that the LPVNS had grown slowly and finally became a multiple nodular form. Although there was no connection between LPVNS and DPVNS, LPVNS should be diagnosed and treated as soon as possible because early resection led to a good prognosis. When there was vague and long lasting knee pain, LPVNS should be considered in the differential diagnosis and after the diagnosis was confirmed, early meticulous arthroscopic synovectomy was helpful for a good outcome.

Currently, there was no standard method for nonsurgically observing LPVNS. The eradication of all aberrant synovial tissue in symptomatic patients was considered a suitable treatment option for LPVNS, as it effectively relieved pain and prevented local recurrence. Nevertheless, the optimal type of resection and whether adjuvant therapy should be used remains controversial. ${ }^{27}$ Open synovectomy was routinely performed for extraarticular extension of LPVNS when full excision cannot be achieved with an arthroscope. ${ }^{20,28}$ However, open excision may lead to postoperative muscle weakness and an increased risk of osteoarthritic changes. With advances in arthroscopy, all arthroscopic total synovectomy had been shown to be equally effective..$^{20,29-31}$ In addition, the combination of the two techniques using a posterior open and anterior arthroscopic approach may be ideal when commonly used portals cannot be used to completely excise the posterior abnormal synovial. ${ }^{32}$ In recent years, some research had suggested that it was necessary to excise some otherwise normal appearing fat and areolar tissue with the synovium in cases of recurrence. ${ }^{33}$ In general, the appropriate extent of excision should be determined on the basis of the type of PVNS, the presence of extraarticular extensions and the experience of the surgeon. In addition, conventional radiotherapy as well as radiosynoviorthesis was considered for patients with large and even recurrent diffuse forms of the disease. ${ }^{34}$ Preoperatively, although the patient had been recommended to receive radiosynoviorthesis 6-8 weeks after surgery, she declined to accept the adjuvant therapy because of the concerns about the potential side effects of the radiopharmacon. Fortunately, both intraoperative findings and the postoperative MRI ensured that no residual PVNS was found. So far, the complete surgical excision without radiation therapy was satisfactory in the present case. Nevertheless, PVNS still had a certain rate of recurrence; in some cases, recurrence occured even after 16 years postoperatively. ${ }^{35}$ The diffuse form of the disease, incomplete resection, the lesions being located in locations difficult to access, the surgeon having limited experience and skills and the administration of adjuvant therapy after surgery have been identified as the major risk factors for recurrence. $^{27}$ Approximately two-thirds of local recurrences were diagnosed within postoperative 2 years, while less than $10 \%$ of recurrences were found after 3 years and it was recommended that follow up MRI scans were performed every 6 months during the first 3 years to detect local recurrences effectively. ${ }^{36}$ In this patient, we successfully performed open complete excision. Symptom relief was achieved and no recurrence was observed during the subsequent follow up; however, the long-term curative effect remains to be assessed (Figure 3).

\section{CONCLUSION}

To reduce the occurrence of misdiagnosis, MRI examinations are recommended for all patients suspected of having PVNS, including those who have a history of hyperuricemia. Early diagnosis with prompt surgical treatment may lead to favourable recovery.

Funding: No funding sources

Conflict of interest: None declared

Ethical approval: Not required

\section{REFERENCES}

1. Murphey MD, Rhee JH, Lewis RB, Fanburg-Smith JC, Flemming DJ, Walker EA. Pigmented villonodular synovitis: radiologic-pathologic correlation. Radiographics. 2008;28(5):1493-518.

2. Ottaviani S, Ayral X, Dougados M, Gossec L. Pigmented villonodular synovitis: a retrospective single-center study of 122 cases and review of the literature. Semin Arthritis Rheum. 2011;40(6):539-46.

3. Shah SH, Porrino JA, Green JR, Chew FS. Bilateral pigmented villonodular synovitis of the knee. Radiol Case Rep. 2015;10(4):56-60.

4. O'Connell JX. Pathology of the synovium. Am J Clin Pathol. 2000;114(5):773-84.

5. Xie GP, Jiang N, Liang CX, Zeng JC, Chen ZY, Xu $\mathrm{Q}$, et al. Pigmented villonodular synovitis: a retrospective multicenter study of 237 cases. PloS One. 2015;10(3):0121451.

6. Abdul-Karim FW,Naggar AK, Joyce MJ, Makley JT, Carter JR. Diffuse and localized tenosynovial giant cell tumor and pigmented villonodular ynovitis: a clinicopathologic and flow cytometric DNA analysis. Human Pathol. 1992;23(7):729-35.

7. Flandry F, Hughston JC. Pigmented villonodular synovitis. J Bone Joint Surg. 1987;69(6):942-9.

8. Flandry F, Hughston JC, McCann SB, Kurtz DM. Diagnostic features of diffuse pigmented villonodular synovitis of the knee. Clinic Orthopaed Rel Res. 1994;298:212-20.

9. Flandry FC, Hughston JC, Jacobson KE, Barrack RL, McCann SB, Kurtz DM. Surgical treatment of diffuse 
pigmented villonodular synovitis of the knee. Clinic Orthopaed Rel Res. 1994;300:183-92.

10. Gu HF, Zhang SJ, Zhao C, Chen Y, Bi Q. A comparison of open and arthroscopic surgery for treatment of diffuse pigmented villonodular synovitis of the knee. Knee Surg Sport Traumatol Arthrosc. 2014;22(11):2830-6.

11. Valle AGD, Piccaluga F, Potter HG, Salvati EA, Pusso R. Pigmented villonodular synovitis of the hip: 2- to 23-year followup study. Clinic Orthopaed Rel Res. 2001;388:187-99.

12. Fecek C, Carter KR. Pigmented villonodular synovitis. Treasure Island (FL): StatPearls Publishing; 2020.

13. Bassetti E, Candreva R, Santucci E. Pigmented villonodular synovitis of the knee: a case report. J Ultrasound. 2011;14(3):167-9.

14. Friedman T, Chen T, Chang A. MRI diagnosis of recurrent pigmented villonodular synovitis following total joint arthroplasty. HSS. 2013;9(1):100-5.

15. Garner HW, Ortiguera CJ, Nakhleh RE. Pigmented villonodular synovitis. Radiographics. 2008;28(5):1519-23.

16. Granowitz SP, Mankin HJ. Localized pigmented villonodular synovitis of the knee. Report of five cases. J Bone Joint Surg Am. 1967;49(1):122-8.

17. Auregan JC, Klouche S, Bohu Y, Lefevre N, Herman S, Hardy P. Treatment of pigmented villonodular synovitis of the knee. Arthroscopy. 2014;30(10):1327-41.

18. Stephan SR, Shallop B, Lackman R, Kim TW, Mulcahey MK. Pigmented villonodular synovitis: a comprehensive review and proposed treatment algorithm. JBJS Rev. 2016;4(7).

19. Dines JS, DeBerardino TM, Wells JL, Dodson CC, Shindle M, DiCarlo EF, et al. Long-term follow-up of surgically treated localized pigmented villonodular synovitis of the knee. Arthroscopy. 2007;23(9):930-7.

20. Ogilvie-Harris DJ, McLean J, Zarnett ME. Pigmented villonodular synovitis of the knee. The results of total arthroscopic synovectomy, partial, arthroscopic synovectomy, and arthroscopic local excision. J Bone Joint Surg Am. 1992;74(1):119-23.

21. Atik OS, Bozkurt HH, Ozcan E, Bahadir B, Ucar M, Ogut B, et al. Localized pigmented villonodular synovitis in a child knee. Joint Dis Rel Surg. 2017;28(1):46-9.

22. Sezgin EA, Atik OS. Tumors and tumor-like lesions of infrapatellar fat pad and surrounding tissues: a review of the literature. Joint Dis Rel Surg. 2018;29(1):58-62.

23. Asik M, Erlap L, Altinel L, Cetik O. Localized pigmented villonodular synovitis of the knee. Arthroscopy. 2001;17(6):23.
24. Calmet J, Hernandez-Hermoso J, Gine J, Jimeno F. Localized pigmented villonodular synovitis in an unusual location in the knee. Arthroscopy. 2003;19(2):144-9.

25. Flandry FC, Jacobson KE, Andrews JR. Localized pigmented villonodular synovitis of the knee mimicking meniscal injury. Arthroscopy. 1986;2(4):217-21.

26. Moraux A, Bianchi S. Soft tissue masses of the knee related to a focal defect of the lateral patellar retinaculum. J Ultrasound Med. 2018;37(7):1821-5.

27. Fang Y, Zhang Q. Recurrence of pigmented villonodular synovitis of the knee: a case report with review of literature on the risk factors causing recurrence. Medicine. 2020;99(16):19856.

28. Chin KR, Barr SJ, Winalski C, Zurakowski D, Brick GW. Treatment of advanced primary and recurrent diffuse pigmented villonodular synovitis of the knee. J Bone Joint Surg Am. 2002;84(12):2192-202.

29. DePonti A, Sansone V, Malchere M. Result of arthroscopic treatment of pigmented villonodular synovitis of the knee. Arthroscopy. 2003;19(6):602-7.

30. Zvijac JE, Lau AC, Hechtman KS, Uribe JW, Tjin ATEW. Arthroscopic treatment of pigmented villonodular synovitis of the knee. Arthroscopy. 1999;15(6):613-7.

31. Papamerkouriou YM, Posantzis MI, Kouremenos D, Manousakis C, Plessas SI. Arthroscopic technique for the treatment of localized pigmented villonodular synovitis of the knee. Cureus. 2020;12(4):7832.

32. Sharma V, Cheng EY. Outcomes after excision of pigmented villonodular synovitis of the knee. Clin Orthop Relat Res. 2009;467(11):2852-8.

33. Jobe CM, Raza A, Zuckerman L. Pigmented villonodular synovitis: extrasynovial recurrence. Arthroscopy. 2011;27(10):1449-51.

34. Dürr HR, Capellen CF, Klein A, Baur-Melnyk A, Birkenmaier C, Jansson V, et al. The effects of radiosynoviorthesis in pigmented villonodular synovitis of the knee. Arch Orthop Trauma Surg. 2019;139(5):623-7.

35. Verspoor FG, Zee AA, Hannink G, Geest IC, Veth RP, Schreuder HW. Long-term follow-up results of primary and recurrent pigmented villonodular synovitis. Rheumatology. 2014;53(11):2063-70.

36. Capellen CF, Tiling R, Klein A, Baur-Melnyk A, Knösel T, Birkenmaier C, et al. Lowering the recurrence rate in pigmented villonodular synovitis: a series of 120 resections. Rheumatology. 2018;57(8):1448-52.

Cite this article as: Juneja J, Choubisa R, Sen R, Mehra AK, Aggarwal B, Sharma KK. Intra and extraarticular localized pigmented villonodular synovitis. Int J Res Orthop 2022;8:110-4. 